\title{
Punicalagin Regulates Key Processes Associated with Atherosclerosis in THP-1 Cellular Model
}

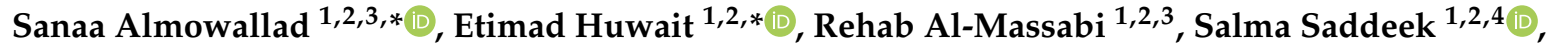 \\ Kalamegam Gauthaman ${ }^{5,6}$ and Alexandre Prola ${ }^{7}$ \\ 1 Department of Biochemistry, Faculty of Sciences, King Abdul Aziz University, Jeddah 21589, Saudi Arabia; \\ rf-saif@ut.edu.sa (R.A.-M.); salmayms@uhb.edu.sa (S.S.) \\ 2 Cell Culture Unit, King Fahad Medical Research Centre, King Abdul Aziz University, Jeddah 22252, \\ Saudi Arabia \\ 3 Department of Biochemistry, Faculty of Sciences, University of Tabuk, Tabuk 71491, Saudi Arabia \\ 4 Department of Chemistry, Faculty of Sciences, University of Hafr Al Batin, Hafr Al Batin 31991, Saudi Arabia \\ 5 Center of Excellence in Genomic Medicine Research, King Abdulaziz University, Jeddah 21589, Saudi Arabia; \\ Kgauthaman@kau.edu.sa \\ 6 Department of Medical Laboratory Technology, Faculty of Applied Medical Sciences, \\ King Abdulaziz University, Jeddah 21589, Saudi Arabia \\ 7 Department of Cell Physiology and Metabolism, Faculty of Medicine, University of Geneva, \\ 1 rue Michel-Servet, CH-1211, 1202 Geneva, Switzerland; alexandre.prola@unige.ch \\ * Correspondence: salmowaled@ut.edu.sa (S.A.); ehuwait@kau.edu.sa (E.H.); \\ Tel.: +966547097545 (S.A.); +966505508255 (E.H.)
}

Received: 20 October 2020; Accepted: 6 November 2020; Published: 8 November 2020

check for updates

\begin{abstract}
Atherosclerosis may lead to cardiovascular diseases (CVD), which are the primary cause of death globally. In addition to conventional therapeutics for CVD, use of nutraceuticals that prevents cholesterol deposition, reduce existing plaques and hence anti-atherosclerotic effects of nutraceuticals appeared to be promising. As such, in the present study we evaluated the beneficial effects of punicalagin, a phytochemical against an atherosclerotic cell model in vitro. Cytotoxicity assays were examined for $10 \mu \mathrm{M}$ concentration of punicalagin on THP-1 macrophages. Real-time-polymerase chain reaction (RT-PCR) was used to analyze monocyte chemoattractant protein-1 (MCP-1) and Intercellular adhesion molecule (ICAM-1) expressions. Monocyte migration and cholesterol efflux assays were performed to investigate punicalagin's further impact on the key steps of atherosclerosis. Cytotoxicity assays demonstrated no significant toxicity for punicalagin $(10 \mu \mathrm{M})$ on THP-1 macrophages. Punicalagin inhibited the IFN- $\gamma$-induced overexpression of MCP-1 and ICAM-1 in macrophages by 10 fold and 3.49 fold, respectively, compared to the control. Punicalagin also reduced the MCP-1- mediated migration of monocytes by $28 \%$ compared to the control. Percentages of cellular cholesterol efflux were enhanced in presence or absence of IFN- $\gamma$ by $88 \%$ and $84 \%$ compared to control with $58 \%$ and $62 \%$, respectively. Punicalagin possesses anti-inflammatory and anti-atherosclerotic effects. Punicalagin also did not exhibit any cytotoxicity and therefore can be considered a safe and potential candidate for the treatment and prevention of atherosclerosis.
\end{abstract}

Keywords: atherosclerosis; punicalagin; inflammation; monocyte migration; cholesterol efflux

\section{Introduction}

Currently, cardiovascular-related diseases (CVD) remain the leading cause of death globally in comparison to other chronic illnesses. In 2012, CVD was responsible for 31\%, or 17.5 million deaths across the globe, and the number of fatalities is estimated to rise to approximately 23.3 million 
within the next decade [1]. Moreover, the increased prevalence of CVD poses considerable burdens for healthcare systems due to the high costs involved in treating and managing the condition $[1,2]$. Atherosclerosis remains the primary cause of deadly heart diseases [3] and occurs when long-lasting inflammation affects the vascular system.

Atherosclerosis is a progressive illness that results from the accumulation of fatty acids and lipids in medium- and large-sized arteries, which then become precursors to the buildup of plaque [4]. Over time, size of plaque increase and may eventually rupture, releasing vast amounts of necrotic fragments into the bloodstream, thus increasing the probability of blood clots leading to myocardial infarctions, stroke, or both [5].

Atherosclerosis treatment includes the administration of statin as a main core element. However, a significant issue with its use concerns increased associations with a continued risk of CVD, as well as causing severe, adverse side effects such as diabetes [6] or hemorrhagic stroke [7] in different patient populations. Current studies examining other pharmaceutical agents for atherosclerosis treatment have, thus far, yielded disappointing results during clinical trials. In this context, the development of nutraceuticals appears to be promising to prevent the development of atherosclerosis. The THP-1 macrophage cell line is thought to be an interesting model for studying the anti-atherosclerotic potential of many natural products $[8,9]$.

Punicalagin is a phytochemical polyphenolic compound extracted from pomegranate (Punica granatum) and is the most abundant polyphenol in pomegranate juice $(80 \% w / w)$ [10]. Punicalagin is known to have strong antioxidant characteristics in comparison to other nutraceuticals [3,11]. Studies on patients undergoing hemodialysis demonstrated that pomegranate juice did not affect or alter inflammatory markers expression after six months of about $1000 \mathrm{mg}$ per day uptake [12]. Another study has reported that cholesterol level and oxidized low-density lipoprotein (LDL) were found to be reduced after consuming pomegranate juice [13]. However, past research has prioritized the effects of pomegranate crude extracts [14] or juice [13] and has not investigated the anti-atherogenic effects of punicalagin alone. It is well-known that pomegranate is a great source of antioxidants and therefore were used for treating heart disease. However, pomegranate has been used traditionally in treating many health conditions such as pancreatic inflammation, vaginal discharge, diabetes, and diarrhea [15]. As a result, the physiological activity of punicalagin on various vital atherosclerosis episodes remains currently poorly understood.

Monocyte chemoattractant protein-1 (MCP-1) is a chemokine known for its ability to recruit monocytes and macrophages to the site of inflammation [16]. Intercellular adhesion molecule-1 (ICAM-1) is involved in contacts with other cells or with the extracellular matrix [17] and enables macrophages to migrate towards inflammation sites [18]. Previous studies suggest that MCP-1 and ICAM-1 are essential markers in several vascular diseases, among which is atherosclerosis, by recruiting monocytes and favoring the adhesion of macrophages to the active endothelium $[5,18,19]$.

The aim of this study was to investigate markers for atherosclerosis, including inflammation and lipid deposition, in response to punicalagin in classically activated type 1 macrophages (M1) differentiated from THP-1 monocytic cell line. In addition, we investigated human monocyte migration and cholesterol efflux to assess the effect of punicalagin on atherosclerosis progression.

\section{Results}

\subsection{Punicalagin Does Not Alter THP-1 Macrophages Viability}

Punicalagin-induced cytotoxicity was measured by lactate dehydrogenase (LDH) assays whereas cell viability was measured by crystal violet using $10 \mu \mathrm{M}$ concentration of punicalagin on THP-1 macrophages. LDH is a cytoplasmic enzyme released in the extracellular medium when plasma membrane is damaged. Therefore, LDH release is commonly used to assess cytotoxicity. As shown in Figure 1a, there was no significant release of LDH by the macrophages following incubation with punicalagin compared to vehicle-treated cells. Crystal violet binds to the DNA of adherent cells; thus, 
the staining correlates with cell number. Detached cells were considered as non-viable and discarded before quantification. Punicalagin treatment induced no significant difference in macrophage viability compared with vehicle-treated cells (Figure 1b). Furthermore, combined findings from crystal violet and LDH assays, $10 \mu \mathrm{M}$ concentration of punicalagin was used for further PCR.

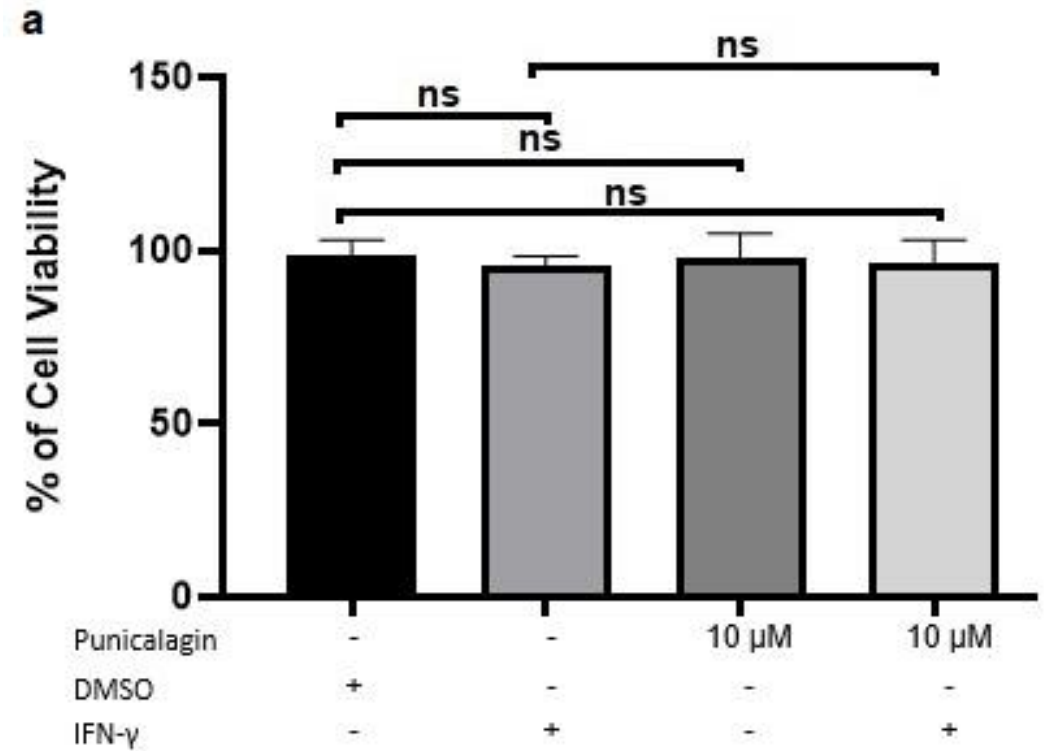

b

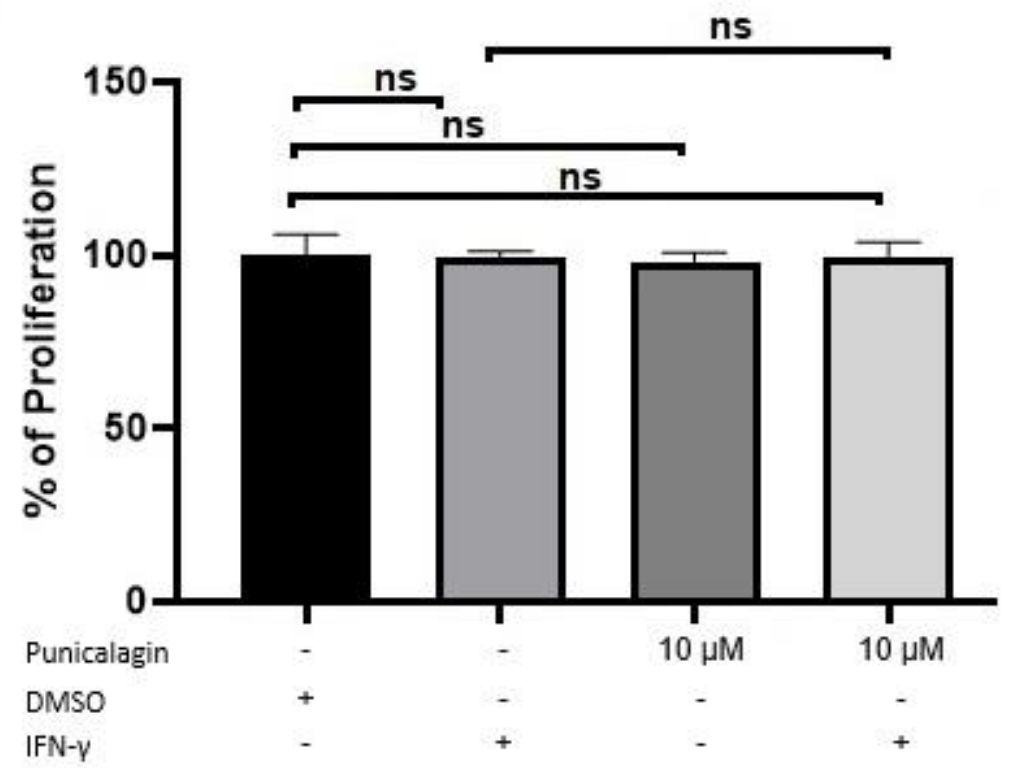

Figure 1. Analysis of punicalagin-induced cytotoxicity on THP-1 macrophages. Treatment of cells with $10 \mu \mathrm{M}$ of punicalagin or dimethyl sulfoxide (DMSO) was conducted for $24 \mathrm{~h}$. Values are represented as mean of three independent experiments +/- SEM. (a) Lactate dehydrogenase release was measured by removing media from the treated plates. (b) Remaining macrophages were used for the crystal violet assay. Values of average percentage of dead cells and cell viability are displayed in comparison to vehicle, which was set at 1 . ns = non-significant.

\subsection{Punicalagin Reduces IFN- $\gamma$-Induced mRNA Overexpression of MCP-1 and ICAM-1 in} THP-1 Macrophages

THP-1 macrophages were incubated for $3 \mathrm{~h}$ with IFN- $\gamma$ to induce the expression of MCP-1 and ICAM-1 [20]. Subsequently, cells were treated for another $24 \mathrm{~h}$ using 5 and $10 \mu \mathrm{M}$ of punicalagin 
or with $0.1 \%$ of dimethyl sulfoxide (DMSO) as control. IFN- $\gamma$ induced an overexpression of MCP-1 by 10 and of ICAM-1 by 3.49 (Figure 2). Treatment using 5 and $10 \mu \mathrm{M}$ of punicalagin resulted in a $38 \%$ and $88 \%$ reduction of IFN- $\gamma$-induced MCP-1 overexpression and in a $79 \%$ and $90 \%$ reduction of IFN- $\gamma$-induced ICAM-1 overexpression, respectively (Figure 2).
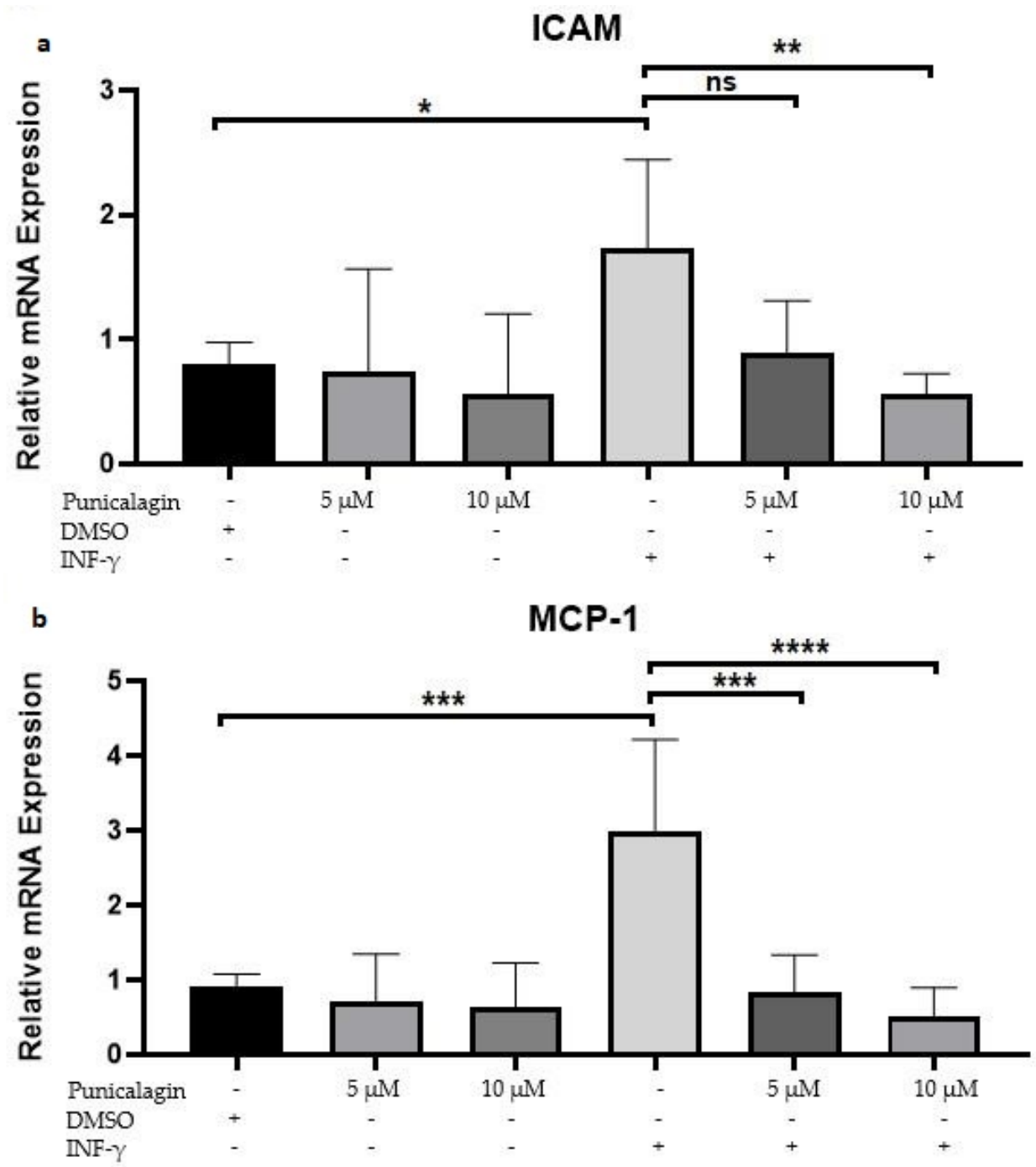

Figure 2. Effect of punicalagin on IFN- $\gamma$-induced MCP-1 and ICAM-1 mRNA levels. Macrophages were incubated for $3 \mathrm{~h}$ with DMSO or IFN $-\gamma$. Cells were treated for $24 \mathrm{~h}$ with punicalagin or DMSO alone. RT-qPCR were performed with specific primers to amplify MCP-1 (a), ICAM-1 (b) and GAPDH complementary DNA (cDNA). The graphs identify the relative fold-changes compared to control, which was assigned to 1 . Values are represented as mean of three independent experiments $+/-$ SEM. $*: p<0.05,{ }^{* *}: p<0.02,{ }^{* * *}: p<0.01,{ }^{* * *}: p<0.001$. ns $=$ non-significant.

\subsection{Punicalagin Inhibits MCP-1-Mediated Migration of THP-1 Monocytes}

During the progression of atherosclerosis, the recruitment of monocytes to the affected area is a crucial mechanism underlying lesion formation. Therefore, we analyzed punicalagin's effect on MCP-1-induced monocytes recruitment. The results of our Boyden chamber experiment, presented in Figure 3, showed a significant increase of 75\% in monocyte migration after MCP-1 addition, compared to control sample $(p<0.0001)$. Interestingly, the presence of punicalagin reduced MCP-1-induced monocyte recruitment by $28 \%(p<0.0001)$. 


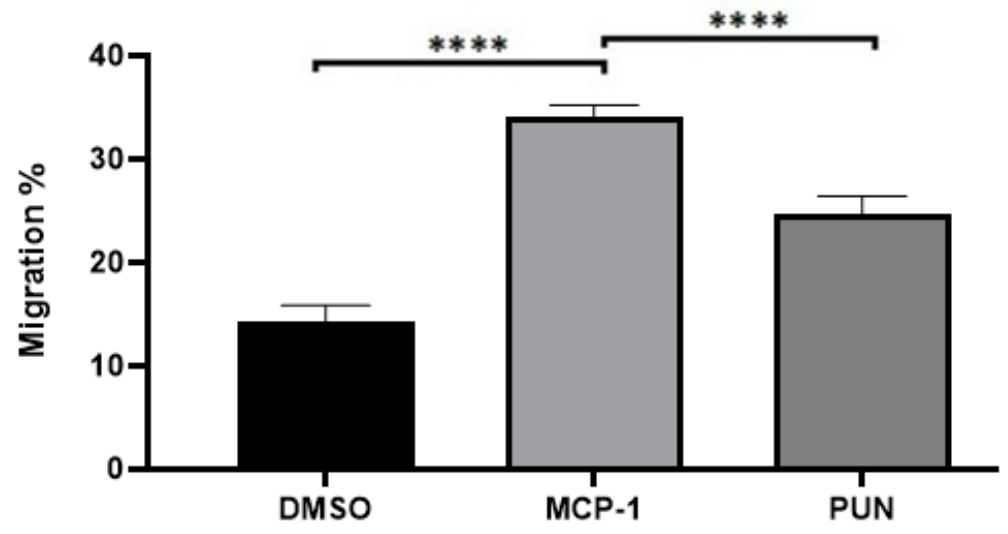

Figure 3. Effect of punicalagin on MCP-1-induced migration of THP-1 monocytes. THP-1 monocytes were treated with DMSO as a vehicle control or with $20 \mathrm{ng} / \mathrm{mL} \mathrm{MCP}-1$ or $20 \mathrm{ng} / \mathrm{mL}$ MCP-1 with $10 \mu \mathrm{M}$ of punicalagin for $3 \mathrm{~h}$. Data were normalized to the percentage of cells that migrated from the apical compartment of a modified Boyden chamber into the basolateral compartment in response to MCP-1 alone. Data are presented as mean \pm SEM following three independent experiments. Statistical analysis was performed using a one-way ANOVA with a Sidak's test analysis where ${ }^{* * * *}: p<0.001$. $\mathrm{PUN}=$ punicalagin.

\subsection{Punicalagin Enhances Cholesterol Efflux in IFN- $\gamma$-Induced THP-1 Macrophages}

Another key step in atherosclerosis management is cellular cholesterol efflux. Punicalagin seems to have a significant enhancement on cellular cholesterol efflux in THP-1 foam cell model. Results from cholesterol efflux assay presented in Figure 4 showed a non-significant increase in cholesterol efflux in cells treated with $10 \mu \mathrm{M} / \mathrm{mL}$ of punicalagin. However, a significant increase in cholesterol efflux was reported for IFN- $\gamma$-induced THP- 1 foam cells treated with $10 \mu \mathrm{M}$ of punicalagin compared to vehicle control. The percentages of cellular cholesterol efflux reported for treatment with $10 \mu \mathrm{M}$ of punicalagin in the presence or absence of IFN- $\gamma$ were $54.2 \%$ and $51.8 \%$, respectively, with comparison to the percentages of cellular cholesterol efflux of vehicle control in presence or absence of IFN- $\gamma$, which are $40 \%$ and $36.6 \%$, respectively.

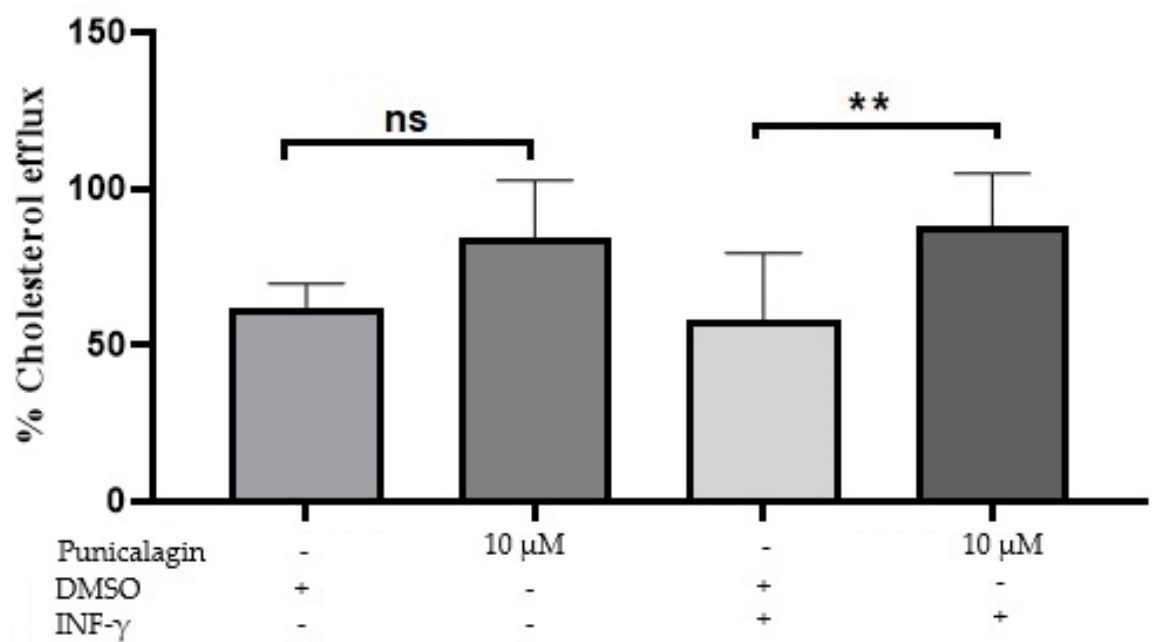

Figure 4. Effect of punicalagin on cholesterol efflux in IFN- $\gamma$-induced THP-1 macrophages. THP-1 differentiated macrophages were incubated with or without of IFN- $\gamma$ for $3 \mathrm{~h}$. THP- 1 cells were labeled with florescent cholesterol and incubated overnight. Treatment with $10 \mu \mathrm{M}$ of punicalagin were added for $24 \mathrm{~h}$. The percentages of cholesterol efflux were then calculated. Data are presented as mean \pm SEM following three independent experiments. Statistical analysis was performed using a one-way ANOVA with a Sidak's test analysis where ${ }^{* *}: p<0.02, \mathrm{~ns}=$ non-significant. 


\section{Discussion}

Although the beneficial effect of pomegranates on cardiovascular disease are well-known, the underlying mechanism is poorly understood. The aim of this study is to examine Punicalagin's anti-atherosclerotic effects on macrophages in vitro. Two key factors leading to atherosclerosis are inflammation [21] or lipid deposition [22]. Based on recent in vivo research, pomegranate has anti-atherosclerotic and anti-inflammatory properties [23]. Our goal in this study is to find out the favored mechanism of action for punicalagin either anti-inflammatory or anti-hyperlipidemic mechanism. We examined the role of punicalagin on pro-inflammatory gene expression and monocyte migration in order to understand punicalagin mechanism of action. Additionally, punicalagin effect on cholesterol efflux process were examined to find out if anti-hyperlipidemic mechanism of action were favored.

Anti-inflammatory effects of pomegranate and/or punicalagin have been widely demonstrated in the literature [24-28]. To study anti-inflammatory effects of punicalagin on THP-1 macrophage, IFN- $\gamma$ induction were used to accelerate several pro-inflammatory gene expressions, in particular ICAM-1 and MCP-1 [20,24], which contribute to the adhesion and conscription of cells in vitro. During this process, IFN- $\gamma$ increases the binding of ICAM- 1 to endothelial cells $[29,30]$. In this context, our results interestingly demonstrated the ability of punicalagin to significantly reduce IFN- $\gamma$-induced expression of ICAM-1 and MCP-1. This result corroborates a previous study that evidenced MCP-1 down-regulation induced by punicalagin in human intestinal cells [11].

This inhibition of IFN- $\gamma$-induced inflammatory signaling by punicalagin is an essential finding, as a default in IFN- $\gamma$ signaling is associated to the diminution of atherosclerosis lesions in vivo [31]. Thus, our results strongly suggest that IFN- $\gamma$ signaling is a central target of the anti-atherogenic and anti-inflammatory actions of punicalagin.

Nevertheless, a question remains regarding the molecular pathway through which punicalagin inhibits IFN- $\gamma$-induced expression of MCP-1 and ICAM-1. It is well-known that antioxidants reduce harmful oxidative stress by reacting with free radicals and subsequently reduce inflammation [32,33]. These findings may be linked to the well-known antioxidant property of punicalagin [34]. Our results are promising and call for the development of further investigations to identify the molecular mechanisms behind the anti-atherosclerotic effects of punicalagin.

Peripheral circulating monocytes migrate into tissues during the inflammatory process as a critical pro-inflammatory response. In atherosclerosis, monocytes are related to hypercholesterolemia-associated migration to inflamed arteries, extravasation, sub-endothelial accumulation, and differentiation into macrophages [35]. MCP-1 is known to promote the migration and infiltration of monocytes/macrophages. In cardiovascular diseases, it has been demonstrated that MPC-1 deficiency results in a substantial reduction in arterial lipid deposition [36]. Our results presented on Figure 3 bring additional knowledge to this field of research, as we demonstrated that punicalagin considerably reduces MCP-1-induced monocyte migration. Taken together, these results could be essential for the prevention and the treatment of atherosclerosis.

On the other hand, anti-hyperlipidemic effect of punicalagin were examined in our study. Previous studies have mentioned the association of cholesterol efflux capacity in relation to atherosclerosis [37]. Accumulation of cholesterol in foam cell macrophages will result in plaque formation that leads to atherosclerosis [38-40]. Our data obtained from cholesterol efflux assay has demonstrated the capacity of punicalagin to enhance cholesterol efflux in IFN- $\gamma$-induced THP-1 macrophages. This will probably give a clue on how punicalagin acts as an anti-atherosclerotic agent.

\section{Materials and Methods}

\subsection{Reagents}

For all experiments, Roswell Park Memorial Institute (RPMI) 1640 culture medium Cat No. A1049101; Fetal bovine serum Cat No. A3160802; Penicillin-Streptomycin Cat No. 15140122; 
2-mercaptoethanol Cat No. M6250-10ML, L-Glutamine (200 mM) Cat No. 25030081, Phosphate buffered saline (PBS) solution Cat No. 10010015 and MCP-1 (MCAF) Recombinant Human Protein Cat No. PHC1014 were obtained from (Gibco-BRL, Cheshire, UK). Punicalagin ( $\geq 98 \%$ HPLC) Cat No. P0023, phorbol myristate acetate (PMA), human interferon-gamma and crystal violet were obtained from (Sigma-Aldrich, Gillingham UK). Dimethyl sulfoxide (DMSO) was obtained from (Invitrogen, Carlsbad, CA, USA). Pierce LDH Cytotoxicity Assay Kit was obtained from (Thermo Fisher Scientific, Waltham, MA, USA). RNeasy Mini Kit Cat No: 74104 and QuantiFast SYBR Green PCR Kit Cat No.: 204054 were obtained from (Qiagen, Manchester, UK). Im Prom-II Reverse Transcription System Cat No: A3800 was obtained from (Promega, Madison, WI, USA).

\subsection{Preparation of Punicalagin Treatment}

Ten mg of punicalagin were diluted in $921 \mu \mathrm{L}$ of DMSO to achieve a concentration of $10 \mathrm{mM}$. This main stock can be stored in the dark at $-20^{\circ} \mathrm{C}$ for at least six months.

\subsection{THP-1 Cell Culture}

THP-1 cells (monocytic leukemia cell lines) were obtained from the Molecular Biomedicine Unit at King Faisal Specialist Hospital and Research Centre (KFSHRC) in Riyadh, Saudi Arabia. Upon receiving the cells, they were sub-cultured in suspension in T75 and/or T25 flasks in a vertical position. RPMI-1640 was supplemented with antibiotics $(100 \mu \mathrm{g} / \mathrm{mL}$ of both streptomycin and penicillin) and $10 \%$ fetal bovine serum (FBS). THP-1 cells were sub-cultured every 48-72 h. Trypan blue assay was used to examine cell viability.

\subsection{Lactate Dehydrogenase (LDH) Assay}

THP-1 monocytes were seeded in 96-well plates at densities of 105 cells $/ \mathrm{cm}^{2}$ and differentiated by supplementing the RPMI medium with $160 \mathrm{nM}$ of PMA. Differentiation of the seeded cells into related macrophages occurred overnight in an incubator at a humidity of $5 \%$ carbon dioxide $v / v$. To initiate foam cell formation, $130 \mathrm{nM}$ of IFN- $\gamma$ were added for $3 \mathrm{~h}$ to one set of the samples and vehicle in the other. Subsequently, the differentiated cells were exposed to $10 \mu \mathrm{M}$ of punicalagin for another $24 \mathrm{~h}$. All cells from the positive control well were lysed using the lysis buffer from the LDH kit for $45 \mathrm{~min}$. A negative control was used for background reading. Using a 96-well plate, $50 \mu \mathrm{L}$ volume of the well's content solution was removed into a new 96 well plate and mixed using $50 \mu \mathrm{L}$ of assay buffer. After $30 \mathrm{~min}$ of incubation at room temperature, $50 \mu \mathrm{L}$ of stopping solution was added. Absorption was read at $570 \mathrm{~nm}$ using a BioTek plate reader (BioTek Instruments, Winooski, VT, USA). The negative values of the control were deducted from actual readings and read as percentage of cell viability in comparison to the experimental control.

\subsection{Crystal Violet Assay}

For the crystal violet assay, we used macrophages from the LDH assay to meet the experimental conditions. Remaining culture media were discarded from the original 96 well plate obtained from LDH assay and $50 \mu \mathrm{L}$ of $0.2 \%(w / v)$ crystal violet solution (containing $10 \%$ ethanol) were added for $5 \mathrm{~min}$ at room temperature to stain the cells. Macrophages were washed at least three times in PBS before adding $50 \mu \mathrm{L}$ of a solubilization buffer (containing $0.1 \mathrm{M} \mathrm{NaH}_{2} \mathrm{PO}_{4}$ dissolved in ethanol). The treated plate was aired on a shaking stand for $5 \mathrm{~min}$ before using a microplate reader at $570 \mathrm{~nm}$ to read its absorbance. The crystal violet bound DNA of adherent cells, that are considered as live cells. Results are expressed as the percentage of viability compared to control conditions.

\subsection{RNA Extraction}

For RNA extraction, we initiated differentiation from monocytes to macrophages by seeding THP-1 monocytes in six T25 flasks and treating all cells with PMA. The differentiation process using 
PMA occurred over $24 \mathrm{~h}$ in an incubator, at a humidity of $5 \%$ carbon dioxide $v / v$. Following incubation, one flask was treated with DMSO only as a control and second one was treated with IFN- $\gamma$ only for $3 \mathrm{~h}$. The four other flasks were treated with 5 or $10 \mu \mathrm{M}$ of punicalagin in the absence or presence of IFN- $\gamma$. Twenty hours later, cells were harvested and RNeasy kit (Qiagen, Valencia, CA, USA) was used to extract RNA according to the manufacturer specifications. RNA concentration was measured using a NanoDrop ND 1000 spectrophotometer (ThermoFisher Scientific, Wilmington, DE, USA), while RNA purity was specified using the A260/A280 ratio. A ratio between 1.8 and 2.1 indicated high-purity RNA.

\subsection{Analysis of Gene Expression Using $q R T-P C R$}

Complementary DNA (cDNA) was prepared via reverse transcription of mRNA using the ImProm-II Reverse Transcription kit (Promega, Madison, WI, USA), according to the manufacturer's protocol. For long-term storage, cDNA products were kept frozen at -80 or $-20{ }^{\circ} \mathrm{C}$. Quantitative PCR was performed using cDNA in a PCR 96-well plate from (Applied Biosystems, Beverly, MA, USA) and the SYBR Green Kit (Qiagen, Manchester, UK) according to the manufacturer's protocol. StepOnePlus real-time-polymerase chain reaction (RT-PCR) System (Applied Biosystems, Waltham, MA, USA) was used to run the PCR.

The genes of interest were MCP-1 and ICAM-1. Glyceraldehyde-3-phosphate dehydrogenase (GAPDH) was used as a reference gene. Primer sequences of genes are as follows in (Table 1):

Table 1. Primer Sequences of Genes Used in RT-qPCR.

\begin{tabular}{ccc}
\hline Genes & Forward Sequence $\left(\mathbf{5}^{\prime} \mathbf{-} \mathbf{3}^{\prime} \mathbf{)}\right.$ & Reverse Sequence $\mathbf{( 5}^{\prime} \mathbf{- 3}^{\prime} \mathbf{)}$ \\
\hline H-GAPDH & CTTTTGCGTCGCCAGCCGAG & GCCCAATACGACCAAATCCGTTGACT \\
H-MCP-1 & CGCTCAGCCAGATGCAATCAATG & ATGGTCTTGAAGATCACAGCTTCTTTGG \\
H-ICAM-1 & GACCAGAGGTTGAACCCCAC & GCGCCGGAAAGCTGTAGAT \\
\hline
\end{tabular}

PCR reactions were completed using the following steps: denaturation occurred at $94{ }^{\circ} \mathrm{C}$ for $120 \mathrm{~s}$, and 40 amplification cycles of denaturation $\left(30 \mathrm{~s}\right.$ at $\left.95^{\circ} \mathrm{C}\right)$ - annealing step $\left(60 \mathrm{~s}\right.$ at $\left.60{ }^{\circ} \mathrm{C}\right)$ - extension step $\left(60 \mathrm{~s}\right.$ at $72^{\circ} \mathrm{C}$ ) [41]. Messenger RNA expression changes of MCP-1 and ICAM-1 were compared to the internal housekeeping control GAPDH and calculated using the $\mathrm{Ct}$ relative gene expression method [42]. The values were analyzed using Microsoft Excel 2010 and GraphPad Prism version 8 software (San Diego, CA, USA).

\subsection{MCP-1-Induced Monocyte Migration Assay}

Monocyte migration in response to MCP-1 was investigated using a Boyden chamber assay as previously described [43]. An insert containing a membrane with $8 \mu \mathrm{m}$ pores was used to split wells into separate halves to mimic the arterial endothelium layer, while allowing monocytes migration. To induce migration, $20 \mathrm{ng} / \mathrm{mL}$ of MCP-1 chemokine or DMSO was added to a total of $1 \mathrm{~mL}$ culture media to the bottom half of all the wells. Undifferentiated THP- 1 monocytes $(5 \times 105$ cells/well $)$ were added to the top half of the well. Culture media, either containing punicalagin or DMSO, was then added to the top halves of the wells until a total volume of $0.5 \mathrm{~mL}$ was achieved. The modified Boyden chambers were then incubated at $37^{\circ} \mathrm{C}$ in $5 \%(v / v) \mathrm{CO} 2$ for $3 \mathrm{~h}$.

The media in the top half of the wells was then removed, and the underside of the membrane washed with $0.5 \mathrm{~mL}$ PBS to remove any adherent cells. The media in the bottom of the wells was then transferred to a $10 \mathrm{~mL}$ Falcon tube and centrifuged at $250 \times \mathrm{g}$ for $5 \mathrm{~min}$ at room temperature. Cells were resuspended in $2 \mathrm{~mL}$ of culture media and counted using a hemocytometer. The number of cells that had migrated from the top to the bottom half of the well was calculated and expressed as a percentage of the number of cells that had been originally added. 


\subsection{Cholesterol Efflux Assay}

To examine the effect of punicalagin on the progression of atherosclerosis in our cellular model, cholesterol efflux was performed using commercial Cholesterol Efflux Assay Kit Cat No.: ab196985 from (Abcam, UK). THP-1 monocytes were counted and $1 \times 10^{5}$ cells/well were seeded in a 96-well black plate with clear bottom using $100 \mu \mathrm{L}$ media/well and incubated for one hour to settle down. Then, 160 nM of PMA were added to allow differentiation into adherent macrophages and cells were incubated overnight. To initiate foam cell formation, $130 \mathrm{nM}$ of IFN- $\gamma$ was added for $3 \mathrm{~h}$ to one set of the samples and vehicle in the other. After incubation, cell monolayer was washed with serum free RPMI 1640 media. Labeling Reagent and Equilibration Buffer (provided in the kit) were mixed just before use. One hundred microliters per well were added to cells. After overnight incubation, Labeling Reagent was removed, and cells were washed gently by adding $200 \mu \mathrm{L}$ of serum free RPMI. One hundred microliters of fresh RPMI containing $10 \mu \mathrm{M}$ punicalagin were added to each well in both sets of samples. Cells were incubated for $24 \mathrm{~h}$ in a $37{ }^{\circ} \mathrm{C}$ incubator containing $5 \% \mathrm{CO}_{2}$.

For wells of positive control, $20 \mu \mathrm{L}$ of Positive Control (provided in the kit) were added to $80 \mu \mathrm{L}$ of RPMI while $100 \mu \mathrm{L}$ of serum free RPMI were added to negative control wells. Cells were incubated for another $4 \mathrm{~h}$ in a $37^{\circ} \mathrm{C}$ incubator containing $5 \% \mathrm{CO}_{2}$. Then, supernatant was transferred to a new 96-well plate while cell monolayer was solubilized by $100 \mu \mathrm{L}$ of cell lysis buffer and put on a plate shaker for $30 \mathrm{~min}$ at room temperature. Fluorescence of the two resulting plates were measured using $\mathrm{Ex} / \mathrm{Em}=482 / 515 \mathrm{~nm}$. The percentage of cholesterol efflux was calculated by dividing the fluorescence intensity of the medium by the total of fluorescence intensity of medium and cell lysate.

$$
\% \text { Cholesterol Efflux }=\frac{\text { Fluorescence Intensity of Media }}{\text { Fluorescence Intensity of Media }+ \text { Cell Lysate }} \times 100
$$

\subsection{Statistical Analysis}

Statistical analysis was performed using a one-way ANOVA followed by a Sidak post-hoc analysis. Software used for statistical analysis were Excel Microsoft 2010 and GraphPad Prism version 8.

\section{Conclusions}

The results obtained during the present study indicate that punicalagin regulates several key processes related to atherosclerosis. These findings are demonstrated by punicalagin's inhibition of IFN- $\gamma$-induced MCP-1 and ICAM-1 gene expression at mRNA level and reduction of MCP-1 mediated monocyte migration and enhancement of cholesterol efflux from macrophages in vitro. Our current results suggest that punicalagin could be of interest to prevent atherosclerosis development and further in vivo research would be important.

Author Contributions: Conceptualization, methodology and supervision, E.H.; primary investigation, visualization, data analysis, and writing-original draft preparation, S.A.; project administration, R.A.-M. and S.S.; writing-review and editing, K.G. and A.P. All authors have read and agreed to the published version of the manuscript.

Funding: We have received no funding for this work.

Acknowledgments: We would like to thank Dipak Ramji, Cardiff University, Cardiff, UK for his invaluable advice, comments, and continuous support throughout. We would also like to thank Khalid Abu Khabar, King Faisal Specialist Hospital \& Research Center (KFSHRC), Riyadh, Saudi Arabia for his generous THP-1 cell line gift, which has been a crucial part of our research.

Conflicts of Interest: The authors declare no conflict of interest. 


\section{References}

1. Buckley, M.L.; Ramji, D.P. The influence of dysfunctional signaling and lipid homeostasis in mediating the inflammatory responses during atherosclerosis. Biochim. Biophys. Acta Mol. Basis Dis. 2015, 1852, 1498-1510. [CrossRef] [PubMed]

2. Oldridge, N.B. Economic burden of physical inactivity: Healthcare costs associated with cardiovascular disease. Eur. J. Prev. Cardiol. 2008, 15, 130-139. [CrossRef] [PubMed]

3. Quirós-Fernández, R.; López-Plaza, B.; Bermejo, L.M.; Palma-Milla, S.; Gómez-Candela, C. Supplementation with hydroxytyrosol and punicalagin improves early atherosclerosis markers involved in the asymptomatic phase of atherosclerosis in the adult population: A randomized, placebo-controlled, crossover trial. Nutrients 2019, 11, 640. [CrossRef] [PubMed]

4. Lusis, A.J. Genetics of atherosclerosis. Trends Genet. 2012, 28, 267-275. [CrossRef]

5. Lusis, A.J. Atherosclerosis-Insight Review Articles. Nature 2000, 407, 233-241. [CrossRef]

6. White, J.; Swerdlow, D.I.; Preiss, D.; Fairhurst-Hunter, Z.; Keating, B.J.; Asselbergs, F.W.; Sattar, N.; Humphries, S.E.; Hingorani, A.D.; Holmes, M.V. Association of lipid fractions with risks for coronary artery disease and diabetes. JAMA Cardiol. 2016, 1, 692-699. [CrossRef]

7. Mihaylova, B.; Emberson, J.; Blackwell, L.; Keech, A.; Simes, J.; Barnes, E.H.; Voysey, M.; Gray, A.; Collins, R.; Baigent, C.; et al. The effects of lowering LDL cholesterol with statin therapy in people at low risk of vascular disease: Meta-analysis of individual data from 27 randomised trials. Lancet 2012, 380, 581-590. [CrossRef] [PubMed]

8. Orekhov, A.N.; Ivanova, E.A. Cellular models of atherosclerosis and their implication for testing natural substances with anti-atherosclerotic potential. Phytomedicine 2016, 23, 1190-1197. [CrossRef]

9. Chanput, W.; Mes, J.J.; Wichers, H.J. THP-1 cell line: An in vitro cell model for immune modulation approach. Int. Immunopharmacol. 2014, 23, 37-45. [CrossRef] [PubMed]

10. Seeram, N.; Lee, R.; Hardy, M.; Heber, D. Rapid large scale purification of ellagitannins from pomegranate husk, a by-product of the commercial juice industry. Sep. Purif. Technol. 2005, 41, 49-55. [CrossRef]

11. Hollebeeck, S.; Winand, J.; Hérent, M.F.; During, A.; Leclercq, J.; Larondelle, Y.; Schneider, Y.J. Anti-inflammatory effects of pomegranate (Punica granatum L.) husk ellagitannins in Caco-2 cells, an in vitro model of human intestine. Food Funct. 2012, 3, 875-885. [CrossRef] [PubMed]

12. Wu, P.T.; Fitschen, P.J.; Kistler, B.M.; Jeong, J.H.; Chung, H.R.; Aviram, M.; Phillips, S.A.; Fernhall, B.; Wilund, K.R. Effects of Pomegranate Extract Supplementation on Cardiovascular Risk Factors and Physical Function in Hemodialysis Patients. J. Med. Food 2015, 18, 941-949. [CrossRef]

13. Aviram, M.; Rosenblat, M. Pomegranate protection against cardiovascular diseases. Evid.-Based Complement. Altern. Med. 2012, 2012. [CrossRef]

14. de Nigris, F.; Williams-Ignarro, S.; Sica, V.; Lerman, L.O.; D'Armiento, F.P.; Byrns, R.E.; Casamassimi, A.; Carpentiero, D.; Schiano, C.; Sumi, D.; et al. Effects of a Pomegranate Fruit Extract rich in punicalagin on oxidation-sensitive genes and eNOS activity at sites of perturbed shear stress and atherogenesis. Cardiovasc. Res. 2007, 73, 414-423. [CrossRef]

15. Lansky, E.; Shubert, S.; Neeman, I. Pharmacological and therapeutic properties of pomegranate. Adv. Res. Technol. 2000, 235, 231-235.

16. Calvo, C.F.; Yoshimura, T.; Gelman, M.; Mallat, M. Production of monocyte chemotactic protein-1 by rat brain macrophages. Eur. J. Neurosci. 1996, 8, 1725-1734. [CrossRef] [PubMed]

17. Wautier, J.; Setiadi, H.; Vilette, D. Leukocyte Adhesion to Endothelial Cells (Accepted by Editor G.W. Schmid-Schonbein). 1990, pp. 425-432. Available online: https://content.iospress.com/articles/biorheology/ bir27-3-4-19 (accessed on 8 November 2020).

18. Muller, W.A. Mechanisms of leukocyte transendothelial migration. Annu. Rev. Pathol. Mech. Dis. 2011, 6, 323-344. [CrossRef]

19. Kitagawa, K.; Matsumoto, M.; Sasaki, T.; Hashimoto, H.; Kuwabara, K.; Ohtsuki, T.; Hori, M. Involvement of ICAM-1 in the progression of atherosclerosis in APOE-knockout mice. Atherosclerosis 2002, 160, 305-310. [CrossRef] 
20. Li, N.; McLaren, J.E.; Michael, D.R.; Clement, M.; Fielding, C.A.; Ramji, D.P. ERK Is Integral to the IFN- $\gamma$-Mediated Activation of STAT1, the Expression of Key Genes Implicated in Atherosclerosis, and the Uptake of Modified Lipoproteins by Human Macrophages. J. Immunol. 2010, 185, 3041-3048. [CrossRef] [PubMed]

21. Mullenix, P.S.; Andersen, C.A.; Starnes, B.W. Atherosclerosis as inflammation. Ann. Vasc. Surg. 2005, 19, 130-138. [CrossRef]

22. Peng, J.; Luo, F.; Ruan, G.; Peng, R.; Li, X. Hypertriglyceridemia and atherosclerosis. Lipids Health Dis. 2017, 16. [CrossRef]

23. Salama, A.A.; Ismael, N.M.; Bedewy, M. The Anti-inflammatory and Antiatherogenic In Vivo Effects of Pomegranate Peel Powder: From Waste to Medicinal Food. J. Med. Food 2020, 1-6. [CrossRef]

24. Jean-Gilles, D.; Li, L.; Vaidyanathan, V.G.; King, R.; Cho, B.; Worthen, D.R.; Chichester, C.O.; Seeram, N.P. Inhibitory effects of polyphenol punicalagin on type-II collagen degradation in vitro and inflammation in vivo. Chem. Biol. Interact. 2013, 205, 90-99. [CrossRef] [PubMed]

25. Lee, C.J.; Chen, L.G.; Liang, W.L.; Wang, C.C. Multiple activities of punica granatum linne against acne vulgaris. Int. J. Mol. Sci. 2017, 18, 141. [CrossRef]

26. Atrahimovich, D.; Samson, A.O.; Khattib, A.; Vaya, J.; Khatib, S. Punicalagin Decreases Serum Glucose Levels and Increases PON1 Activity and HDL Anti-Inflammatory Values in Balb/c Mice Fed a High-Fat Diet. Oxid. Med. Cell. Longev. 2018, 2018. [CrossRef]

27. Yaidikar, L.; Thakur, S. Punicalagin attenuated cerebral ischemia-reperfusion insult via inhibition of proinflammatory cytokines, up-regulation of Bcl-2, down-regulation of Bax, and caspase-3. Mol. Cell. Biochem. 2015, 402, 141-148. [CrossRef]

28. Peng, J.; Wei, D.; Fu, Z.; Li, D.; Tan, Y.; Xu, T.; Zhou, J.; Zhang, T. Punicalagin Ameliorates Lipopolysaccharide-Induced Acute Respiratory Distress Syndrome in Mice. Inflammation 2015, 38, 493-499. [CrossRef]

29. Rimbach, G.; Valacchi, G.; Canali, R.; Virgili, F. Macrophages stimulated with IFN- $\gamma$ activate NF- $k b$ and induce MCP-1 gene expression in primary human endothelial cells. Mol. Cell Biol. Res. Commun. 2000, 3 , 238-242. [CrossRef]

30. Chang, Y.J.; Holtzman, M.J.; Chen, C.C. Interferon- $\gamma$-induced epithelial ICAM-1 expression and monocyte adhesion. Involvement of protein kinase c-dependent c-Src tyrosine kinase activation pathway. J. Biol. Chem. 2002, 277, 7118-7126. [CrossRef] [PubMed]

31. Gupta, S.; Tall, A.R.; Schindler, C. IFN-gamma potentiates atherosclerosis in ApoE knock-out mice. J. Clin. Investig. 1997, 99, 2752-2761. [CrossRef]

32. Lobo, V.; Patil, A.; Phatak, A.; Chandra, N. Free radicals, antioxidants and functional foods: Impact on human health. Pharmacogn. Rev. 2010, 4, 118-126. [CrossRef]

33. Arulselvan, P.; Fard, M.T.; Tan, W.S.; Gothai, S.; Fakurazi, S.; Norhaizan, M.E.; Kumar, S.S. Role of Antioxidants and Natural Products in Inflammation. Oxid. Med. Cell. Longev. 2016, 2016. [CrossRef]

34. Gil, M.I.; Tomas-Barberan, F.A.; Hess-Pierce, B.; Holcroft, D.M.; Kader, A.A. Antioxidant activity of pomegranate juice and its relationship with phenolic composition and processing. J. Agric. Food Chem. 2000, 48, 4581-4589. [CrossRef]

35. Mestas, J.; Ley, K. Monocyte-Endothelial Cell Interactions in the Development of Atherosclerosis. Trends Cardiovasc. Med. 2008, 18, 228-232. [CrossRef]

36. Boring, L.; Gosling, J.; Cleary, M.; Charo, I.F. Decreased lesion formation in CCR2(-/-) mice reveals a role for chemokines in the initiation of atherosclerosis. Nature 1998, 394, 894-897. [CrossRef]

37. Khera, A.V.; Cuchel, M.; de la Llera-Moya, M.; Rodrigues, A.; Burke, M.F.; Jafri, K.; French, B.C.; Phillips, J.A.; Mucksavage, M.L.; Wilensky, R.L.; et al. Cholesterol Efflux Capacity, High-Density Lipoprotein Function, and Atherosclerosis. N. Engl. J. Med. 2012, 2012, 206-208. [CrossRef]

38. Moore, K.J.; Sheedy, F.J.; Fisher, E.A. Macrophages in atherosclerosis: A dynamic balance. Nat. Rev. Immunol. 2013, 13, 709-721. [CrossRef] [PubMed]

39. Volobueva, A.; Zhang, D.; Grechko, A.V.; Orekhov, A.N. Foam cell formation and cholesterol trafficking and metabolism disturbances in atherosclerosis. Cor Vasa 2019, 61, E48-E54. [CrossRef]

40. Yu, X.H.; Fu, Y.C.; Zhang, D.W.; Yin, K.; Tang, C.K. Foam cells in atherosclerosis. Clin. Chim. Acta 2013, 424, 245-252. [CrossRef] [PubMed] 
41. Moss, J.W.E.; Davies, T.S.; Garaiova, I.; Plummer, S.F.; Michael, D.R.; Ramji, D.P. A unique combination of nutritionally active ingredients can prevent several key processes associated with atherosclerosis in vitro. PLoS ONE 2016, 11, e0151057. [CrossRef]

42. Livak, K.J.; Schmittgen, T.D. Analysis of relative gene expression data using real-time quantitative PCR and the 2- $\Delta \Delta$ CT method. Methods 2001, 25, 402-408. [CrossRef] [PubMed]

43. Gosling, J.; Slaymaker, S.; Gu, L.; Tseng, S.; Zlot, C.H.; Young, S.G.; Rollins, B.J.; Charo, I.F. MCP-1 deficiency reduces susceptibility to atherosclerosis in mice that overexpress human apolipoprotein B. J. Clin. Investig. 1999, 103, 773-778. [CrossRef]

Publisher's Note: MDPI stays neutral with regard to jurisdictional claims in published maps and institutional affiliations.

(C) 2020 by the authors. Licensee MDPI, Basel, Switzerland. This article is an open access article distributed under the terms and conditions of the Creative Commons Attribution (CC BY) license (http://creativecommons.org/licenses/by/4.0/). 